\title{
Chromosomal inversions effect body size and shape in different breeding resources in Drosophila buzzatii
}

\author{
PJ Fernández Iriarte ${ }^{1,2}$, FM Norry ${ }^{2,3}$ and ER Hasson ${ }^{2}$ \\ ${ }^{1}$ Laboratorio de Genética, Departamento de Biología, Facultad de Ciencias Exactas y Naturales, Universidad Nacional de Mar del Plata, \\ Funes 3250, 7600 Mar del Plata, Buenos Aires, Argentina; ${ }^{2}$ GIBE, Departamento de Ecología, Genética y Evolución, Facultad de Ciencias \\ Exactas y Naturales, Universidad de Buenos Aires, Ciudad Universitaria Pab. II, EHA1428 Buenos Aires, Argentina; ${ }^{3}$ Department of \\ Ecology and Genetics, University of Aarhus, Ny Munkegade, Bldg. 540, DK-8000 Aarhus C, Denmark
}

The cactophilic Drosophila buzzatii provides an excellent model for the study of reaction norms across discrete environments because it breeds on rotting tissues (rots) of very different cactus species. Here we test the possible effects of second chromosome inversions on body size and shape (wing loading) across suitable natural breeding substrates. Using homokaryotypic stocks derived from several lines homozygous for four naturally occurring chromosomal inversions, we show that arrangements significantly affect size-related traits and wing loading. In addition, karyotypes show differing effects, across natural breeding resources, for wing loading. The $2 \mathrm{st}$ and $2 \mathrm{jz} \mathrm{z}^{3}$ arrangements decrease and the $2 \mathrm{j}$ arrangement increases wing loading. For thorax length and wing loading, karyotypic correlations across host plants are slightly lower in females than in males. These results support the hypothesis that these traits have a genetic basis associated with the inversion polymorphism.

Heredity (2003) 91, 51-59. doi:10.1038/sj.hdy.6800280

Keywords: chromosomal inversion; Drosophila; body size; wing loading; cactus hosts

\section{Introduction}

Several studies have shown that inversion polymorphisms in Drosophila affect size-related traits (Ruiz et al, 1991; Hasson et al, 1992a) and body shape (Bitner-Mathé et al, 1995). The main interest in the linking of these chromosomal polymorphisms to phenotypic variation is that this may provide a mechanistic approach for investigating the causes of natural selection acting on these widespread polymorphisms.

There is an important body of evidence showing that body size is correlated with fecundity (Robertson, 1957), dispersal ability (Roff, 1977), mating success (Partridge et al, 1987; Santos et al, 1988), longevity (Santos et al, 1992; Hasson et al, 1993; Rodríguez et al, 1999; Reeve et al, 2000; Norry and Loeschcke, 2002) and development time (Partridge and Fowler, 1993; Betrán et al, 1998; Fernández Iriarte and Hasson, 2000). Other studies have shown that body size (Thomas and Barker, 1993; Nunney and Cheung, 1997), wing shape (Cavicchi et al, 1991; BitnerMathé and Klaczko, 1999) and a composite trait related to flight ability, wing loading (Stalker, 1980), respond to environmental variation in complex ways (Loeschcke et al, 1999), suggesting that reaction norms in these traits may be part of an adaptive exophenotypic response in Drosophila.

Correspondence: PJ Fernández Iriarte, Departament de Genètica $i$ de Microbiologia, Facultat de Ciències, Universitat Autónoma de Barcelona, 08193 Bellaterra, Barcelona, Spain. E-mail: Pedro.Fernandez@uab.es

Received 11 June 2002; accepted 11 February 2003
The cactophilic species Drosophila buzzatii provides an excellent model for the study of reaction norms across discrete environments because it breeds on rotting tissues (rots) of very different cactus species that coexist within a given locality (Hasson et al, 1992b). Rots of different cactus species may differ in yeast diversity (Starmer, 1981), chemical composition (Kircher, 1982) and other ecologically relevant variables such as size and relative density (Hasson et al, 1992b). Likewise, adaptive strategies in cactophilic Drosophila may be related to the temporal and spatial predictability of different cactus species (Etges, 1993; Fanara and Hasson, 2001). Given the potential importance of the maintenance of the genetic variation in heterogeneous environments, it is of considerable interest to test whether or not particular genotypes respond differentially to these discrete environments.

Inversion polymorphisms in D. buzzatii vary clinally along latitudinal and altitudinal gradients that are correlated with geographic variation in temperature (Hasson et al, 1995; Rodríguez et al, 2000). Moreover, second chromosome inversions in this species have been shown to affect thorax length (Ruiz et al, 1991; Hasson et al, 1992a; Norry et al, 1995b), longevity (Hasson et al, 1991; Rodríguez et al, 1999), developmental time and preadult viability (Betrán et al, 1998; Fernández Iriarte and Hasson, 2000). More surprisingly, the effects of polymorphic inversions on the latter two traits strongly depend on the cactus species where flies develop from egg to adult (Fernández Iriarte and Hasson, 2000). Here, we test the possible effects of second chromosome 
inversions on body size and shape across different natural breeding resources.

\section{Materials and methods}

Flies were collected in the natural population of Otamendi (Buenos Aires Province), where D. buzzatii breeds and feeds on the necrotic cladodes of Opuntia vulgaris (see Hasson et al (1995) for a detailed description of the site of collection). This population is polymorphic for four second chromosome inversions namely: st (0.157), j (0.697), jz $\mathrm{z}^{3}(0.142)$ and $\mathrm{jq}^{7}$ (0.004) (number of chromosomes analyzed: 816).

After six to 10 generations of sib-mating, three homozygous lines of each arrangement were used to set up four homokaryotypic stocks (see below), in order to avoid differing levels of consanguinity among stocks. Four experimental stocks homozygous for each arrangement (st/st, j/j, jz $\mathrm{z}^{3} / \mathrm{jz}^{3}$ and $\mathrm{jq}^{7} / \mathrm{jq}^{7}$ ) were established one generation before the experiment (see details in Fernández Iriarte and Hasson, 2000). A total of 100 5- to 6-dayold virgin flies of each sex and desired karyotype were placed in egg-collecting chambers in order to derive all 10 possible karyotypes (st/st, st $/ \mathrm{j}, \mathrm{st} / \mathrm{jz}^{3}$, st $/ \mathrm{jq}^{7}, \mathrm{j} / \mathrm{j}, \mathrm{j} / \mathrm{jz}^{3}$, $\mathrm{j} / \mathrm{jq}^{7}, \mathrm{jz}^{3} / \mathrm{jz}^{3}, \mathrm{jz}^{3} / \mathrm{jq}^{7}$ and $\left.\mathrm{jq}^{7} / \mathrm{jq}^{7}\right)$. Flies were allowed to mate and oviposit for $48 \mathrm{~h}$ in plastic boxes containing Petri dishes with an egg laying medium (15 g agar, $75 \mathrm{ml}$ ethanol (95\%), $15 \mathrm{ml}$ glacial acetic acid in $1500 \mathrm{ml}$ water). Dehydrated Baker's yeast was used to stimulate oviposition. Large numbers of eggs were collected and then sterilized with $50 \%$ clorox for 3 min (Starmer and Gilbert, 1982). Eggs were allowed to hatch, and sets of 40 axenic first-instar larvae were transferred to culture vials containing $6 \mathrm{~g}$ of three different types of cactus media, prepared with fresh tissues of O. vulgaris $(\mathrm{Ov}), \mathrm{O}$. ficusindica (Of) or T. terschekii $(\mathrm{Tt})$. Ov is the host plant in the population from which the flies were derived and constitutes a suboptimal resource (Fernández Iriarte et al, 1999). Of is the main host plant in colonized populations of the Old World (Ruiz et al, 1986) and in some Argentinian populations (Fanara et al, 1996) and its higher amount of carbohydrates could positively affect adult fitness (Brazner et al, 1984). Although D. buzzatii is mainly an Opuntia breeder, it can also breed on necrotic stems of various species of columnar cacti of the genera Trichocereus and Cereus in North Western Argentina (Hasson et al, 1992b). Thus, we also included cardón, Trichocereus terschekii $(\mathrm{Tt})$, as a representative of columnar cacti which, in general, are thought to have a more complex chemical composition that may affect the microflora (yeasts and bacteria) associated with the necrotic pads (Starmer, 1981; Kircher, 1982). D. buzzatii use the rotting stems of $T$. terschekii as breeding and feeding sites in nature (Hasson et al, 1992b). Estimates of viability of $D$. buzzatti flies in media prepared with rotting tissues of cardón (93\%) were similar to those obtained in Of and Ov (90 and 89\%, respectively) (Fernández Iriarte and Hasson, 2000). Therefore, $\mathrm{Tt}$ was considered as a suitable cactus host for D. buzzatii.

Pieces of cactus were ground into a blender and $6 \mathrm{~g}$ poured into standard vials $(2.5 \times 12 \mathrm{~cm})$. Vials were then autoclaved and after cooling each one was inoculated with $0.1 \mathrm{ml}$ of fermenting juice obtained from naturally occurring rots of the corresponding cactus species. The fermenting juice of each cactus was obtained from several natural rots maintained by adding $10 \mathrm{~g}$ of fresh cactus every week. In total, 40 first-instar larvae were transferred to vials $24 \mathrm{~h}$ after the addition of the fermenting juice. Five replicated vials were set up per cactus medium and karyotype. Heterokaryotypic experimental individuals were obtained from both reciprocal crosses in equal proportions. Egg collecting chambers and vials were incubated at $25^{\circ} \mathrm{C}$.

For each karyotypic class, 25 males and 25 females reared in each cactus medium (five flies of each sex from each replicate vial) were scored for the following sizerelated traits: thorax length (TL), wing length (WL), wing width (WW), head width (HW), and face width (FW) (see Norry et al (1995a) for details). We also analyzed, as an estimate of shape, the thorax/wing length ratio (TL/WL) which is directly related to the wing loading (but see Thomas and Barker, 1993; Loeschcke et al, 1999). All measurements were performed with a Wild microscope fitted with an ocular micrometer.

\section{Statistical analysis}

All traits were $\log _{(\mathrm{e})}$ - transformed prior to analyses. ANOVAs were performed separately for each sex and trait, using karyotype and cactus species as fixed factors and replicate (vial) as a random factor nested within each fixed factor.

Genetic variability in plasticity was evaluated first in terms of genotype-by-environment $(\mathrm{G} \times \mathrm{E})$ interaction (ie, karyotype $\times$ cactus interaction) by plotting mean values of the second chromosome karyotypes across cactus hosts. Second, we estimated the correlation between pairs of traits in different environments (Falconer and Mackay, 1996). Karyotypic correlations (Spearman rank) and confidence interval (Zar, 1984) were estimated by using the mean value of each karyotype for each trait in each cactus medium (Fernández Iriarte and Hasson, 2000). The first method provides a test of magnitude of $\mathrm{G} \times \mathrm{E}$ interaction and the last method provides information on the direction of the interaction. A correlation of 0.8 or less indicates a substantial difference in the direction of response with biological significance' (Robertson, 1959).

The linear contribution of each inversion arrangement to the phenotypic value of each trait was tested in each breeding substrate by means of linear regressions on chromosome dosage (Ruiz et al, 1991). For each arrangement we consider the following classes (or chromosome dosage): homozygotes (chromosome dosage 2), heterozygous individuals carrying the gene order under consideration (chromosome dosage 1) and individuals not carrying the arrangement under consideration (chromosome dosage 0 ). This procedure allows the partition of total phenotypic variance into two components of karyotypic variance: additive, given by the proportion of variance explained by the regression $\left(R^{2}\right)$, and nonadditive (dominance plus epistasis), given by the proportion of variation not explained by linear regression.

Since chromosome dosage for a given arrangement is a linear combination of the remaining ones we used the all-subsets methods to find the best reduced model, the one with the largest $R^{2}$ coefficient in the multiple regression. 
Table 1 Two-way ANOVAs performed to test for karyotypic effects on size-related traits and TL/WL scored in flies reared in three different cactus media

\begin{tabular}{|c|c|c|c|c|c|c|}
\hline \multirow[t]{2}{*}{ Trait } & \multirow[t]{2}{*}{ Source } & \multirow[t]{2}{*}{$d f$} & \multicolumn{2}{|c|}{ Males } & \multicolumn{2}{|c|}{ Females } \\
\hline & & & $M S$ & $F$ & $M S$ & $F$ \\
\hline \multirow[t]{5}{*}{ TL } & Karyotype & 9 & 0.0156 & $9.35^{* * *}$ & 0.0140 & $7.77^{* * *}$ \\
\hline & Cactus & 2 & 0.2255 & $135.27^{* * *}$ & 0.3290 & $182.14^{* * *}$ \\
\hline & Replicate & 120 & 0.0016 & $1.85^{* * *}$ & 0.0018 & $1.74^{* * *}$ \\
\hline & Karyotype $\times$ Cactus & 18 & 0.0018 & 1.07 & 0.0027 & 1.52 \\
\hline & Within & 600 & 0.0009 & & 0.0010 & \\
\hline \multirow[t]{5}{*}{ WL } & Karyotype & 9 & 0.0091 & $11.11^{* * *}$ & 0.0074 & $6.41^{* * *}$ \\
\hline & Cactus & 2 & 0.1600 & $196.07^{* * *}$ & 0.2048 & $177.50^{* * *}$ \\
\hline & Replicate & 120 & 0.0008 & $1.46^{* *}$ & 0.0012 & $1.44^{* *}$ \\
\hline & Karyotype $\times$ Cactus & 18 & 0.0010 & 1.24 & 0.0017 & 1.47 \\
\hline & Within & 600 & 0.0006 & & 0.0008 & \\
\hline \multirow[t]{5}{*}{ WW } & Karyotype & 9 & 0.0093 & $9.98^{* * * *}$ & 0.0110 & $8.47^{* * * *}$ \\
\hline & Cactus & 2 & 0.0486 & $52.46^{* * *}$ & 0.0984 & $76.10^{* * *}$ \\
\hline & Replicate & 120 & 0.0009 & $1.42^{* *}$ & 0.0013 & $1.47^{* *}$ \\
\hline & Karyotype $\times$ Cactus & 18 & 0.0012 & 1.26 & 0.0025 & $1.97^{*}$ \\
\hline & Within & 600 & 0.0007 & & 0.0009 & \\
\hline \multirow[t]{5}{*}{ HW } & Karyotype & 9 & 0.0085 & $7.30^{* * *}$ & 0.0105 & $8.64^{* * *}$ \\
\hline & Cactus & 2 & 0.3329 & $285.71^{* * *}$ & 0.3325 & $273.26^{* * *}$ \\
\hline & Replicate & 120 & 0.0012 & $1.74^{* * *}$ & 0.0012 & $1.49^{* *}$ \\
\hline & Karyotype $\times$ Cactus & 18 & 0.0013 & 1.16 & 0.0018 & 1.45 \\
\hline & Within & 600 & 0.0007 & & 0.0008 & \\
\hline \multirow[t]{5}{*}{ FW } & Karyotype & 9 & 0.0456 & $24.15^{* * *}$ & 0.0410 & $18.82^{* * *}$ \\
\hline & Cactus & 2 & 0.2712 & $143.69^{* * *}$ & 0.3914 & $179.69^{* * * *}$ \\
\hline & Replicate & 120 & 0.0019 & $1.45^{* *}$ & 0.0022 & $1.56^{* * *}$ \\
\hline & Karyotype $\times$ Cactus & 18 & 0.0036 & $1.92^{*}$ & 0.0041 & $1.88^{*}$ \\
\hline & Within & 600 & 0.0013 & & 0.0014 & \\
\hline \multirow[t]{5}{*}{ TL/WL } & Karyotype & 9 & 0.0050 & $4.57^{* * *}$ & 0.0030 & $3.95^{* * *}$ \\
\hline & Cactus & 2 & 0.0290 & $28.31^{* * *}$ & 0.0280 & $38.40^{* * *}$ \\
\hline & Replicate & 120 & 0.0010 & $1.57^{* * *}$ & 0.0007 & 1.25 \\
\hline & Karyotype $\times$ Cactus & 18 & 0.0007 & 0.75 & 0.0009 & 1.37 \\
\hline & Within & 600 & 0.0006 & & 0.0006 & \\
\hline
\end{tabular}

${ }^{* * *} P<0.001,{ }^{* *} P<0.01,{ }^{*} P<0.05$.

\section{Results}

In the Appendix, we show the means and standard deviations of size and shape traits for males and females in each cactus host. In O. ficus-indica, D. buzzatii express the biggest size and increase wing loading, whereas in O. vulgaris and T. terschekii there is not a clear trend. On one hand, in the former D. buzzatii express intermediate values for TL, HW and TL/WL ratio while in T. terschekii intermediate values are observed for WW, WL and FW. In general males and females show comparable trends.

Both cactus species and karyotype affect significantly all size-related traits and wing loading, indicating that overall body size and shape are affected, not only by the cactus where the flies developed but also by the chromosomal inversions. Significant karyotype-by-cactus interactions are found only for WW in females and FW in both sexes (Table 1).

There is considerable variation among karyotypes in each cactus host in both sexes, which is most pronounced for wing loading (TL/WL index) (see the Appendix). Reaction norms of the different second chromosome karyotypes across the cactus host is plotted for wing loading (Figure 1). Individuals with homokaryotype st/ st and those with heterokaryotype st/jz $\mathrm{z}^{3}$ and $\mathrm{st} / \mathrm{jq}^{7}$ exhibit lowest wing loading in both sexes in $O$. ficusindica, while individuals $\mathrm{jz}^{3} / \mathrm{jz}^{3}$ shows lowest scores in males reared in O. vulgaris and T. terscheckii. On the other hand, $\mathrm{j} / \mathrm{j}$ and $\mathrm{j} / \mathrm{jq}^{7}$ karyotypes exhibit highest values of wing loading in both sexes in O. ficus-indica.

Karyotypic correlations and the corresponding confidence intervals for each trait between pairs of cactus hosts in males and females are shown in Table 2. In general, positive and significant correlations across hosts $\left(r_{\mathrm{s}}\right.$ differ from zero) are observed for most size-related and shape traits, except for six out of 18 values in males and for nine out of 18 values in females, though, confidence intervals are, in general, large and very close to one. However, it should be noted that the correlations across hosts for the TL/WL ratio in females are very low and different from one.

Karyotypic correlations (using the average in the three cactus media) among size-related traits are positive and most of them significant in both males and females (Table 3). Correlations among size-related traits and shape (TL/WL ratio) are positive but only significant for TL in both sexes and FW only in males.

Regression analysis of each trait on inversion dosage (Table 4) show that $2 \mathrm{jq}^{7}$ affects positively and consistently all size-related traits in both sexes and in all cactus 
media, while it does not seem to affect the TL/WL ratio. Arrangement $2 \mathrm{j}$ also shows a size increasing effect for some traits (mainly TL) only in males and a positive
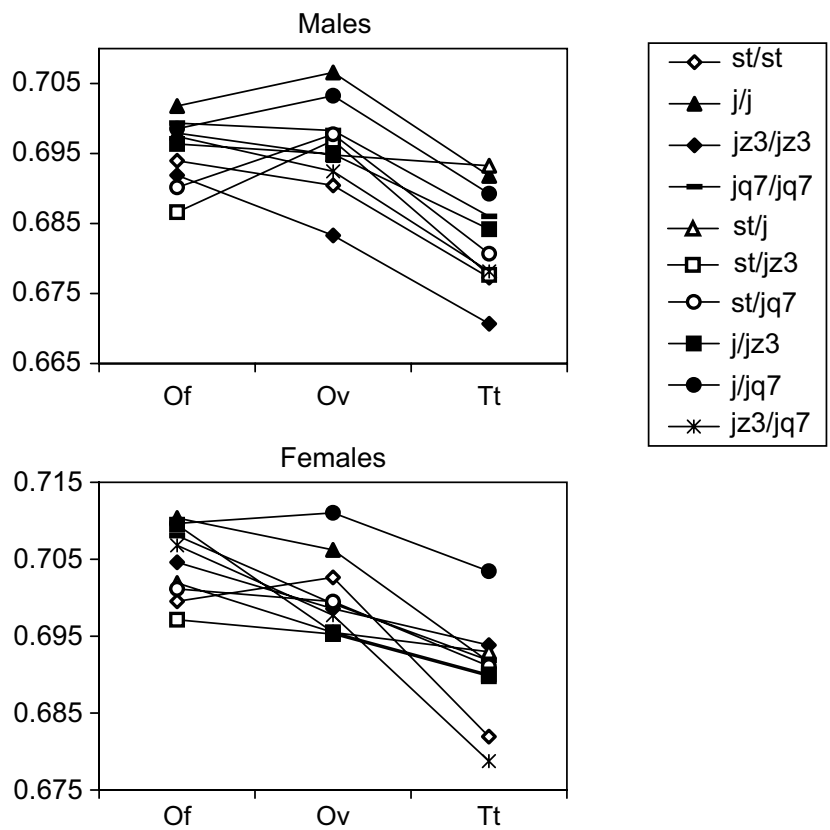

Figure 1 Mean values for each second chromosome karyotypes for TL/WL index in each cactus host (Of: Opuntia ficus-indica; OV: Opuntia vulgaris; Tt: Trichocereus terschekii). effect on TL/WL index in T. terschekii (both sexes) and in O. ficus-indica (males). Inversion $2 \mathrm{jz}^{3}$ shows negative effects on some traits but not in all cactus species and a negative effect on TL/WL index in $O$. vulgaris in both sexes. Arrangement 2st exhibits a negative effect on both WL in males reared in T. terschekii and HW in females reared in $O$. vulgaris and a negative effect on TL/WL index in females reared in O. ficus-indica (Table 4). The proportion of additive variance explained by chromosome inversions (ie, $R^{2}$ for our regression model, see above) is generally higher in both Opuntias cacti (Of and $\mathrm{Ov}$ ) than in cardón ( $\mathrm{Tt}$ ) (Table 4).

\section{Discussion}

The main findings of the present study are that polymorphic second chromosome inversions affect body size and shape (wing loading) in D. buzzatii and that variation in wing loading across natural breeding substrates has a genetic basis that can be partially explained by the inversion polymorphism.

The observed general effects on size and shape (wing loading) depend on the media where flies were reared. D. buzzatti flies emerging on Opuntia ficus-indica were larger than those reared in T. terschekii and O. vulgaris. This may be related to a more restricted supply of nutrients in O. vulgaris (Fernández Iriarte et al, 1999) and T. terschekii. Thus, the nutritional quality of the different breeding sites may drastically affect size and shape traits, as shown by Starmer and Wolf (1989) for wing loading in D. mulleri, which decreased in response to increased

Table 2 Karyotypic correlations between cactus host

\begin{tabular}{|c|c|c|c|c|c|c|}
\hline & & $O f-O v$ & & $O f-T t$ & & $O v-T t$ \\
\hline \multicolumn{7}{|l|}{ Males } \\
\hline $\mathrm{TL}$ & & $0.79(0.95,0.30)$ & & $0.56(0.88,-0.14)$ & & $0.81(0.96,0.35)$ \\
\hline WL & & $0.85(0.97,0.47)$ & & $0.60(0.90,-0.07)$ & & $0.58(0.89,-0.10)$ \\
\hline WW & & $0.90(0.98,0.61)$ & & $0.71(0.93,0.12)$ & & $0.77(0.95,0.25)$ \\
\hline HW & & $0.82(0.96,0.37)$ & & $0.58(0.89,-0.10)$ & & $0.43(0.84,-0.29)$ \\
\hline FW & & $0.79(0.95,0.31)$ & & $0.75(0.94,0.21)$ & & $0.88(0.97,0.54)$ \\
\hline TL/WL & & $0.55(0.88,-0.14)$ & & $0.76(0.94,0.29)$ & & $0.72(0.93,0.15)$ \\
\hline \multicolumn{7}{|l|}{ Females } \\
\hline TL & & $0.64(0.91,-0.01)$ & & $0.52(0.87,-0.19)$ & & $0.41(0.83,-0.31)$ \\
\hline WL & & $0.66(0.92,0.04)$ & & $0.67(0.92,0.05)$ & & $0.65(0.91,0.004)$ \\
\hline WW & & $0.52(0.87,-0.19)$ & & $0.75(0.94,0.21)$ & & $0.60(0.90,-0.07)$ \\
\hline HW & & $0.74(0.98,0.19)$ & & $0.58(0.89,-0.10)$ & & $0.75(0.94,0.21)$ \\
\hline FW & & $0.90(0.98,0.60)$ & & $0.78(0.95,0.28)$ & & $0.87(0.97,0.52)$ \\
\hline TL/WL & & $0.30(0.79,-0.43)$ & & $0.32(0.80,-0.45)$ & & $0.30(0.79,-0.43)$ \\
\hline \multicolumn{7}{|c|}{$\begin{array}{l}\text { Confidence interval are given in parentheses. Of, Opuntia ficus-indica; Ov, Opuntia vulgaris; Tt, Trichocereus terschekii. Bold-face values are } \\
\text { different from zero }(P<0.05) \text {. }\end{array}$} \\
\hline & $T L$ & $W L$ & $W W$ & $H W$ & $F W$ & $T L / W L$ \\
\hline $\mathrm{TL}$ & & $0.68^{*}$ & $0.82^{* *}$ & $0.93^{* * *}$ & $0.93^{* * *}$ & $0.68^{*}$ \\
\hline WL & $0.74^{*}$ & & $0.77^{* *}$ & $0.84^{* *}$ & $0.82^{* *}$ & 0.09 \\
\hline WW & $0.89^{* * *}$ & $0.74^{*}$ & & $0.87^{* *}$ & $0.88^{* * *}$ & 0.52 \\
\hline HW & $0.90^{* * *}$ & $0.75^{*}$ & $0.94^{* * *}$ & & $0.96^{* * *}$ & 0.47 \\
\hline FW & $0.84^{* *}$ & 0.52 & $0.89^{* * *}$ & $0.89^{* * *}$ & & 0.50 \\
\hline TL/WL & $0.68^{*}$ & 0.07 & 0.51 & 0.52 & $0.66^{*}$ & \\
\hline
\end{tabular}

${ }^{* * *} P<0.001,{ }^{* *} P<0.01,{ }^{*} P<0.05$ 
Table 4 Partial regression coefficients $\left(\beta^{\prime}\right)$ and statistics $\left(R^{2}\right.$ and $\left.\mathrm{F}\right)$ of overall multiple regression are shown for regressions of size-related traits and TL/WL on the dosage of each second chromosome arrangement in each cactus medium

\begin{tabular}{|c|c|c|c|c|c|c|c|}
\hline \multirow[t]{2}{*}{ Trait } & \multirow[t]{2}{*}{ Cactus } & \multicolumn{4}{|c|}{$\beta^{\prime}$} & \multirow[t]{2}{*}{$R^{2}$} & \multirow[t]{2}{*}{$F$} \\
\hline & & st & $j$ & $j z^{3}$ & $j q^{7}$ & & \\
\hline \multirow[t]{3}{*}{ TL(M) } & Of & & & & $0.34^{* * *}$ & 0.13 & $12.46^{* * * *}$ \\
\hline & $\mathrm{Ov}$ & & $0.24^{* * *}$ & & $0.35^{* * *}$ & 0.12 & $17.82^{* * * *}$ \\
\hline & $\mathrm{Tt}$ & & $0.33^{* * *}$ & & $0.23^{* * *}$ & 0.11 & $15.61^{* * * *}$ \\
\hline \multirow[t]{3}{*}{ TL(F) } & Of & & $0.13^{*}$ & & $0.38^{* * *}$ & 0.17 & $16.91^{* * *}$ \\
\hline & $\mathrm{Ov}$ & & & $-0.27^{* * *}$ & $0.22^{* * *}$ & 0.15 & $23.78^{* * *}$ \\
\hline & $\mathrm{Tt}$ & & & & $0.21^{* * *}$ & 0.04 & $5.51^{* *}$ \\
\hline \multirow{3}{*}{ WL(M) } & Of & & & & $0.40^{* * *}$ & 0.14 & $20.74^{* * *}$ \\
\hline & $\mathrm{Ov}$ & & & & $0.27^{* * *}$ & 0.07 & $19.55^{* * *}$ \\
\hline & $\mathrm{Tt}$ & $-0.16^{*}$ & & & & 0.03 & $3.28^{*}$ \\
\hline \multirow[t]{3}{*}{ WL(F) } & Of & & & & $0.31^{* * *}$ & 0.10 & $27.05^{* * *}$ \\
\hline & $\mathrm{Ov}$ & & & $-0.23^{* * *}$ & $0.26^{* * *}$ & 0.16 & $23.90^{* * *}$ \\
\hline & $\mathrm{Tt}$ & & & & $0.19 * *$ & 0.04 & $9.15^{* *}$ \\
\hline \multirow[t]{3}{*}{ WW(M) } & Of & & & & $0.33^{* * *}$ & 0.11 & $29.82^{* * *}$ \\
\hline & $\mathrm{Ov}$ & & & & $0.32^{* * *}$ & 0.13 & $19.58^{* * *}$ \\
\hline & $\mathrm{Tt}$ & & $0.17^{* * *}$ & & $0.26^{* * *}$ & 0.07 & $8.86^{* * *}$ \\
\hline \multirow[t]{3}{*}{ WW(F) } & Of & & & & $0.37^{* * *}$ & 0.12 & $17.38^{* * *}$ \\
\hline & $\mathrm{Ov}$ & & & $-0.25^{* * *}$ & $0.32^{* * *}$ & 0.21 & $33.54^{* * *}$ \\
\hline & $\mathrm{Tt}$ & & & & $0.20^{* *}$ & 0.04 & $4.73^{* *}$ \\
\hline \multirow[t]{3}{*}{ HW(M) } & Of & & $0.14^{*}$ & & $0.42^{* * *}$ & 0.16 & $23.17^{* * * *}$ \\
\hline & $\mathrm{Ov}$ & & $0.22^{* * *}$ & & $0.33^{* * *}$ & 0.10 & $33.54^{* * *}$ \\
\hline & $\mathrm{Tt}$ & & & $-0.18^{* * *}$ & & 0.03 & $4.73^{* * *}$ \\
\hline \multirow[t]{3}{*}{$\mathrm{HW}(\mathrm{F})$} & Of & & & & $0.51^{* * *}$ & 0.23 & $37.44^{* * *}$ \\
\hline & $\mathrm{Ov}$ & $-0.16^{*}$ & & $-0.33^{* * *}$ & $0.15^{* * *}$ & 0.16 & $14.98^{* * *}$ \\
\hline & $\mathrm{Tt}$ & & & & $0.17^{* *}$ & 0.03 & $3.89^{*}$ \\
\hline \multirow[t]{3}{*}{ FW(M) } & Of & & & $-0.28^{* * *}$ & $0.39^{* * *}$ & 0.32 & $38.27^{* * *}$ \\
\hline & $\mathrm{Ov}$ & & & & $0.61^{* * *}$ & 0.34 & $65.14^{* * *}$ \\
\hline & $\mathrm{Tt}$ & & $0.28^{* *}$ & & $0.39^{* * *}$ & 0.16 & $23.48^{* * *}$ \\
\hline \multirow[t]{3}{*}{$\mathrm{FW}(\mathrm{H})$} & Of & & & $-0.24^{* * *}$ & $0.45^{* * *}$ & 0.33 & $60.68^{* * *}$ \\
\hline & $\mathrm{Ov}$ & & & & $0.62^{* * *}$ & 0.30 & $35.90^{* * *}$ \\
\hline & $\mathrm{Tt}$ & & & $-0.26^{* * *}$ & & 0.11 & $9.97^{* * *}$ \\
\hline \multirow{3}{*}{ TL/WL(M) } & Of & & $0.19^{* *}$ & & & 0.04 & $4.79^{* * *}$ \\
\hline & $\mathrm{Ov}$ & & & $-0.22^{* *}$ & & 0.07 & $7.68^{* * *}$ \\
\hline & $\mathrm{Tt}$ & & $0.25^{* * *}$ & & & 0.10 & $9.95^{* * *}$ \\
\hline \multirow[t]{3}{*}{ TL/WL(F) } & Of & $-0.25^{* * *}$ & & & & 0.05 & $7.35^{* * *}$ \\
\hline & $\mathrm{Ov}$ & & & $-0.15^{*}$ & & 0.02 & $3.08^{*}$ \\
\hline & $\mathrm{Tt}$ & & $0.18^{* *}$ & & & 0.02 & $3.65^{*}$ \\
\hline
\end{tabular}

M, males; F, females. Of, Opuntia ficus-indica; Ov, Opuntia vulgaris; Tt, Trichocereus terschekii. ${ }^{* * *} P<0.001,{ }^{* *} P<0.01,{ }^{*} P<0.05$.

larval density. In a previous paper we have shown that when $D$. buzzatti is reared on $O$. ficus-indica, flies have a shorter developmental time than on O. vulgaris and on $T$. terschekii (Fernández Iriarte and Hasson, 2000). In this sense, an inferior nutritional quality may be the cause of the extended developmental time and the small wing loading of flies emerging from vials with seminatural media prepared with Ov and Tt.

Genetic variability in plasticity among cactus species is not a significant component of variation for size-related traits, but we have shown that this component was significant for life-history traits such as developmental time and viability (Fernández Iriarte and Hasson, 2000). Although FW showed a significant karyotype $x$ cactus interaction, the correlations estimated for this trait among environments (cactus hosts) were not different from one. These results suggests that the same set of genes affect variation in size-related traits in different cactus hosts. However, in females the wing loading showed a slightly different picture since karyotypic correlations among environments were different from one. These observations suggest that in females different sets of loci may affect variation in wing loading across cactus hosts.
Wing loading is related to flight cost and is probably an important target of natural selection in Drosophila (Stalker, 1980; Starmer and Wolf, 1989; Barker and Krebs, 1995; Azevedo et al, 1998; Loeschcke et al, 1999). Moreover, it has been shown that variation in this compound trait has a significant genetic basis (Azevedo et al, 1998). Likewise, according with past directional selection on wing loading, the heritability of this trait in Drosophila buzzatii is lower than at least one of its components (TL) (Loeschcke et al, 1999). Evidence of a $G \times E$ interaction for wing loading was detected when flies developed at different temperatures in D. buzzatii (Thomas and Barker, 1993; Loeschcke et al, 1999) and D. melanogaster (David et al, 1994). Similarly, temperatures plus nutritional constraints were also shown to affect wing loading in $D$. aldrichi and D. buzzatii (Barker and Krebs, 1995). In the latter study, the reaction norm of wing loading was due to the compound effect of temperature and nutritional quality (Barker and Krebs, 1995). Thus, available evidence suggest that reduced wing loading may be a response to limitations of food supply that may cause strong competition among larvae (Starmer and Wolf, 1989). Therefore, differences in nutritional quality may be a plausible explanation for the wing loading 
variation among cactus hosts detected in the present study.

The effect of inversions on shape (measured as wing loading) is a new finding for the chromosomal polymorphism of D. buzzatii. Reaction norms of karyotypes differ in the direction of response across cacti for wing loading (Figure 1 and Tables 2 and 4). For instance, arrangements 2 st and $2 \mathrm{jz}^{3}$ showed a decreasing effect on wing loading in both Opuntias, whereas $2 \mathrm{j}$ affected positively this trait mainly in T. terschekii.

In $D$. buzzatii natural populations frequencies variation of arrangements 2 st and $2 \mathrm{j}$ are, respectively, positively and negatively correlated with temperature in Australia (Knibb and Barker, 1988) and 2st is negatively correlated with latitude and with altitude in South American populations (Hasson et al, 1995; Rodríguez et al, 2000). In addition, there is evidence that wing loading decreases linearly with latitude and that reduced wing loading has an adaptive advantage for flying at cold temperatures (Stalker, 1980; David et al, 1994). Thus we may ask whether clinal variation of inversion frequencies along latitudinal and thermal gradients can be accounted for by the effect of inversions on wing loading. However 2st, an arrangement that decreases wing loading, has a negative correlation with latitude, exactly the opposite to that expected.

Wing loading can be also related to dispersal costs and dispersal distances (Starmer and Wolf, 1989). Thus, lower values appear to occur among forms that must fly relatively longer distances to find breeding and feeding locations and higher wing loading might be selectively advantageous if shorter flight (hovering) is important at warm temperatures (Starmer and Wolf (1989) and citations therein). Thus, the small wing loading detected for 2 st is expected to confer a better flight performance in natural populations where resources are rare, or more scattered, such as the rotting stems of T. terschekii in the arid zones of South America. The high viability (Fernández Iriarte and Hasson, 2000) and the low wing loading detected for 2st, the ancestral gene order in $D$. buzzatii (Ruiz and Wasserman, 1993), in a cactus host that belongs to the group of putative ancestral host plants of the D. buzzatti cluster (Hasson et al, 1992b), are, perhaps, aspects related to the evolutionary history of host plant utilization in this species. Thus, the ancestral gene order characterized by its small wing loading may be better adapted to exploit a host plant characterized by a patchy and scattered distribution. On the other hand, derived arrangements such as $2 \mathrm{j}$ with their relatively large wing loading, may be advantageous in exploiting more predictable resources such as Opuntias. Moreover, this characteristic may be a plausible explanation for the success of an inversion that has increased in frequency in relatively recent times (Cáceres et al, 2001) probably following the expansion of prickly pears.

Genetic variation in the ratio TL/WL is primarily due to TL (significant positive karyotypic correlation, Table 3). Thus, selection to decrease wing loading will decrease TL, but with not correlated selection for WL as reported by Thomas and Barker (1993). However, Loeschcke et al (1999) observed another picture since variation in wing loading was apparently due to variation in distal wing length (not considered in our studies) but they found also that wing loading has a marginally significant correlation with thorax length. Since these traits could vary along a latitudinal cline (Barker and Krebs, 1995; Azevedo et al, 1998) the patterns of genetic correlations between size and shape traits could be different among populations.

Regression analysis show that both $2 \mathrm{jq}^{7}$ and $2 \mathrm{j}$ increase the size of adult flies in general agreement with previous studies (Ruiz et al, 1991; Hasson et al, 1992a; Norry et al, 1995b; Fernández Iriarte and Hasson, 2000), but only the former had a consistent effect in all cactus hosts (Table 4). Conversely, inversions 2st and $2 \mathrm{jz}^{3}$ decreased size. However, it is important to note that in other studies it has been shown that the latter has a positive effect on body size (Ruiz et al, 1991; Hasson et al, 1992a) which along with the present results suggest that the effects of $2 \mathrm{jz}$ on size-related traits may depend on the rearing medium, that is, a genotype by environment interaction not detected previously. Perhaps, the most interesting observation is the strong effect observed for $2 \mathrm{jq}^{7}$ on all traits studied. This inversion is either rare or absent in natural populations of the New World (Hasson et al, 1995) but increased substantially in frequency during the colonization of the Old World (Fontdevila, 1989). The finding that $2 \mathrm{jq}^{7}$ strongly increases body size in different breeding resources is particularly interesting because this inversion is known to extend developmental time in all natural breeding resources tested (Fernández Iriarte and Hasson, 2000).

Karyotypic correlations of TL/WL index across host plants are slightly smaller in females than in males suggesting that males have a weaker response than females to environmental variation. Similar findings have been reported in D. melanogaster for several morphological traits (Cowley and Atchley, 1990) and for body weight among flies raised with different larval foods (Hillesheim and Stearns, 1991). A similarly asymmetrical response of the sexes, measured as wing asymmetry, has been reported in D. buzzatii populations exposed to temperature stress, suggesting that females are less tolerant to stress than males (Loeschcke et al, 1994).

We have presented here new evidence concerning the effects of the adaptive inversion polymorphism in $D$. buzzatii; however, the characterization of QTLs associated to size and shape traits, mapping on the second chromosome of D. buzzatii, would allow a better understanding of the complex interaction among the exophenotype, the genotype and fitness associated with the inversion polymorphism in Drosophila.

\section{Acknowledgements}

We thank M Santos for helpful discussions and constructive comments on an earlier draft of this manuscript. We thank two anonymous reviewers for valuable suggestions. This work was supported by Universidad de Buenos Aires, CONICET and Universidad Nacional de Mar del Plata grants awarded to EH. PFI is fellow and $\mathrm{FN}$ and $\mathrm{EH}$ are members of Carrera del Investigador Científico of CONICET (Argentina).

\section{References}

Azevedo RBR, James AC, McCabe J, Partridge L (1998). Latitudinal variation of wing: thorax ratio and wing-aspect ratio in Drosophila melanogaster. Evolution 52: 1353-1362. 
Barker JSF, Krebs RA (1995). Genetic variation and plasticity of thorax length and wing lenth in Drosophila aldrichi and D. buzzatii. J Evol Biol 8: 689-709.

Betrán E, Santos M, Ruiz A (1998). Antagonistic pleiotropic effect of second-chromosome inversions on body size and early lifehistory traits in Drosophila buzzatii. Evolution 52: 144-154.

Bitner-Mathé BC, Klaczko LB (1999). Heritability, phenotypic and genetic correlations of size and shape of Drosophila mediopunctata wings. Heredity 3: 688-696.

Bitner-Mathé BC, Pexioto AA, Klaczko LB (1995). Morphological variation in a natural population of Drosophila mediopunctata: altitudinal cline, temporal changes, and influence of chromosomal inversions. Heredity 75: 54-61.

Brazner J, Aberdeen V, Starmer WT (1984). Host-plant shifts and adult survival in the cactus breeding Drosophila mojavensis. Ecol Entomol 9: 375-381.

Cáceres M, Puig M, Ruiz A (2001). Molecular characterization of two natural hotspots in the Drosophila buzzatii genome induced by transposon insertions. Genome Res 11: 1353-1364.

Cavicchi S, Giorgi G, Natali V, Guerra D (1991). Temperaturerelated divergence in experimental populations of Drosophila melanogaster. III. Fourier and centroid analysis of wing shape and relationship between shape variation and fitness. I Evol Biol 4: 141-159.

Cowley DE, Atchley WR (1990). Development and quantitative genetics of correlation structure among body parts of Drosophila melanogaster. Am Nat 135: 242-268.

David JR, Moreteau B, Gauthier JP, Pétavy G, Stockel A, Imasheva AG (1994). Reaction norms of size characters in relation to growth temperature in Drosophila melanogaster: an isofemale lines analysis. Genet Sel Evol 26: 229-251.

Etges WJ (1993). Genetics of host-cactus response and lifehistory evolution among ancestral and derived populations of cactophilic Drosophila mojavensis. Evolution 47: 750-767.

Falconer DS, Mackay TF (1996). Introduction to Quantitative Genetics. 4th edn. Longman: Essex, England.

Fanara JJ, Hasson E (2001). Oviposition acceptance and fecundity schedule in the cactophilic sibling species Drosophila buzzatii and D. koepferae on their natural hosts. Evolution 55: 2615-2619.

Fanara JJ, Hasson E, Rodríguez C, Santos M, Fontdevila A (1996). Are Opuntia hosts a selective factor for the inversion polymorphism of Drosophila buzzatii? Heredity 77: 500-508.

Fernández Iriarte P, Hasson E (2000). The role of the use of different host plants in the maintenance of the inversion polymorphism in the cactophilic Drosophila buzzatii. Evolution 54: 1295-1302.

Fernández Iriarte P, Levy E, Devincenzi D, Fanara JJ, Rodríguez C, Hasson E (1999). Temporal and spatial variation of the inversion polymorphism in two natural population of Drosophila buzzatii. Hereditas 131: 93-99.

Fontdevila A (1989). Founder effects in colonizing populations. The case of Drosophila buzzatii. In: Fontdevila A (ed) Evolutionary Biology of Transient Unstable Populations, Springer-Verlag: Heidelberg. pp 74-95.

Hasson E, Fanara JJ, Rodríguez C, Vilardi JC, Reig OA, Fontdevila A (1992a). The evolutionary history of Drosophila buzzatii. XXIV. Second chromosome inversions have different average effect on thorax length. Heredity 68: 557-563.

Hasson E, Naveira H, Fontdevila A (1992b). The breeding sites of the Argentinian species of the Drosophila mulleri complex (subgenus Drosophila-repleta group). Rev. Chil Hist Nat 65: 319-326.

Hasson E, Fanara JJ, Rodríguez C, Vilardi JC, Reig O, Fontdevila A (1993). The evolutionary history of Drosophila buzzatii. XXVII. Thorax length is positively correlated with longevity in a natural population from Argentina. Genetica 92: 61-65.

Hasson E, Rodríguez C, Fanara JJ, Naveira H, Reig O, Fontdevila A (1995). The evolutionary history of Drosophila buzzatii. XXVI. Macrogeographic patterns in the inversion polymorphisms in New World populations. J Evol Biol 8: 369-384.
Hasson E, Vilardi JC, Naveira H, Fanara JJ, Rodríguez C, Reig O et al (1991). The evolutionary history of Drosophila buzzatii. XVI. Fitness components analysis in a natural population from Argentina. J Evol Biol 4: 209-225.

Hillesheim E, Stearns SC (1991). The responses of Drosophila melanogaster to artificial selection on body weight and its phenotypic plasticity in two larval food environments. Evolution 45: 1909-1923.

Kircher HW (1982). Chemical composition and its relationship to Sonoran desert Drosophila. In: Barker JSF, Starmer WT (eds) Ecological Genetics and Evolution. The Cactus-Yeast-Drosophila Model System, Academic Press: Sydney. pp 143-158.

Knibb WR, Barker JSF (1988). Polymorphic inversion and esterase loci complex on chromosome 2 of Drosophila buzzatii. II. Spatial variation. Aust J Biol Sci 41: 239-246.

Loeschcke V, Bungaard J, Barker JSF (1999). Reaction norms across and genetic parameters at different temperatures for thorax and wing size traits in Drosophila aldrichi and $D$. buzzatii. I Evol Biol 12: 605-623.

Loeschcke V, Krebs RA, Barker JSF (1994). Genetic variation for resistance and acclimation to high temperature stress in Drosophila buzzatii. Biol J Linn Soc 52: 83-92.

Norry FM, Loeschcke V (2002). Temperature induced shifts in association of longevity with body size in Drosophila melanogaster. Evolution 56: 299-306.

Norry FM, Vilardi JC, Fanara JJ, Hasson E (1995a). Courtship success and multivariate analysis of sexual selection on morphometric traits in Drosophila buzzatii (Diptera: Drosophilidae). J Insect Behav 8: 219-229

Norry FM, Vilardi JC, Fanara JJ, Rodríguez C, Hasson E (1995b). An adaptive chromosomal polymorphism affecting sizerelated traits and longevity selection in a natural population of Drosophila buzzatii. Genetica 96: 285-291.

Nunney L, Cheung W (1997). The effect of temperature on body size and fecundity in female Drosophila melanogaster: evidence for adaptive plasticity. Evolution 51: 1529-1535.

Partridge L, Fowler K (1993). Direct and correlated responses to selection on thorax length in Drosophila melanogaster. Evolution 47: 213-226.

Partridge L, Hoffman A, Jones JC (1987). Male size and mating success in Drosophila melanogaster and D. pseudoobscura under field conditions. Anim Behav 35: 468-476.

Reeve MW, Fowler K, Partridge L (2000). Increased body size confers greater fitness at lower experimental temperature in male Drosophila melanogaster. J Evol Biol 13: 836-844.

Robertson A (1959). The sampling variance of the genetic correlation coefficient. Biometrics 15: 469-485.

Robertson FW (1957). Studies in quantitative inheritance XI. Genetic and environmental correlation between body size and egg production in Drosophila melanogaster. J Genet 55: 428-443.

Rodríguez C, Fanara JJ, Hasson E (1999). Inversion polymorphism, longevity, and body size in a natural population of Drosophila buzzatii. Evolution 53: 612-620.

Rodríguez C, Piccinali R, Levy E, Hasson E (2000). Contrasting population genetic structures using allozymes and the inversion polymorphism in Drosophila buzzatii. J Evol Biol 13: 976-984.

Roff D (1977). Dispersal in dipterans: its costs and consequences. J Anim Ecol 46: 443-456.

Ruiz A, Wasserman M (1993). Evolutionary cytogenetics of the Drosophila buzzatii species complex. Heredity 70: 582-596.

Ruiz A, Fontdevila A, Santos M, Seoane M, Torroja E (1986). The evolutionary history of Drosophila buzzatii. VIII. Evidence for endocyclic selection acting on the inversion polymorphism in a natural population. Evolution 40: 740-755.

Ruiz A, Santos M, Barbadilla A, Quezada-Díaz JE, Hasson E, Fontdevila A (1991). Genetic variance for body size in a natural population of Drosophila buzzatii. Genetics 128: 739-750.

Santos M, Ruiz A, Barbadilla A, Quesada-Díaz JE, Hasson E, Fontdevila A (1988). The evolutionary history of Drosophila 
buzzatii. XIV. Larger flies mate more often in nature. Heredity 61: 255-262.

Santos M, Ruiz A, Quesada-Díaz JE, Barbadilla A, Fontdevila A (1992). The evolutionary history of Drosophila buzzatii. XX. Positive phenotypic covariance between field adult fitness components and body size. J Evol Biol 5: 403-422.

Stalker HD (1980). Chromosome studies in wild populations of Drosophila melanogaster. II. Relationship of inversion frequencies to latitude, season, wing-loading and flight activity. Genetics 95: 211-223.

Starmer WT (1981). A comparison of Drosophila habitats according to the physiological attributes of the associated yeast communities. Evolution 35: 35-52.

Starmer WT, Gilbert DG (1982). A quick and reliable method for sterilizing eggs. Drosoph Inf Serv 58: 170-171.
Starmer WT, Wolf LL (1989). Causes of variation in wing loading among Drosophila species. Biol J. Linn Soc 37: 247-261.

Thomas RH, Barker JSF (1993). Quantitative genetic analysis of the body size and shape of Drosophila buzzatii. Theor Appl Genet 85: 598-608.

Zar JH (1984). Biostatistical Analysis, 2nd edn. Prentice-Hall, Inc.: Engelwood Cliffs, NJ.

\section{Appendix}

The mean of size-related traits and shape of Drosophila buzzatii second chromosome karyotypes in each cactus medium is summarized in Table 5. 
Table 5 Mean of size-related traits and shape (in $\mathrm{mm}$ ) of Drosophila buzzatti second chromosome karyotypes in each cactus medium

\begin{tabular}{|c|c|c|c|c|c|c|c|c|c|c|c|c|}
\hline Trait & Cactus & $s t / s t$ & $j / j$ & $j z^{3} / j z^{3}$ & $j q^{7} / j q^{7}$ & $s t / j$ & $s t / j z^{3}$ & $s t / j q^{7}$ & $j / j z^{3}$ & $j / j q^{7}$ & $j z^{3} / j q^{7}$ & Total \\
\hline \multicolumn{13}{|c|}{ (a) Males } \\
\hline \multirow[t]{3}{*}{ TL } & Of & $0.991(0.033)$ & $1.000(0.024)$ & $0.991(0.026)$ & $1.019(0.028)$ & $0.988(0.024)$ & $0.981(0.033)$ & $1.013(0.024)$ & $1.011(0.029)$ & $1.015(0.035)$ & $1.013(0.027)$ & $1.002(0.031)$ \\
\hline & $\mathrm{Ov}$ & $0.941(0.038)$ & $0.959(0.027)$ & $0.939(0.022)$ & $0.965(0.031)$ & $0.941(0.032)$ & $0.944(0.027)$ & $0.971(0.035)$ & $0.956(0.031)$ & $0.992(0.029)$ & $0.949(0.038)$ & $0.956(0.034)$ \\
\hline & $\mathrm{Tt}$ & $0.932(0.014)$ & $0.959(0.015)$ & $0.919(0.012)$ & $0.947(0.014)$ & $0.947(0.015)$ & $0.939(0.013)$ & $0.955(0.012)$ & $0.965(0.015)$ & $0.973(0.013)$ & $0.947(0.015)$ & $0.948(0.036)$ \\
\hline \multirow[t]{3}{*}{ WL } & Of & $1.428(0.029)$ & $1.425(0.030)$ & $1.432(0.026)$ & $1.457(0.025)$ & $1.416(0.026$ & $1.429(0.034)$ & $1.468(0.028)$ & $1.451(0.033)$ & $1.454(0.035)$ & $1.452(0.026)$ & $1.441(0.033)$ \\
\hline & $\mathrm{Ov}$ & $1.363(0.031)$ & $1.357(0.032)$ & $1.374(0.030)$ & $1.382(0.043)$ & $1.355(0.029)$ & $1.354(0.032)$ & $1.391(0.029)$ & $1.377(0.040)$ & $1.410(0.036)$ & $1.371(0.039)$ & $1.373(0.038)$ \\
\hline & $\mathrm{Tt}$ & $1.376(0.032)$ & $1.386(0.031)$ & $1.370(0.040)$ & $1.381(0.041)$ & $1.366(0.030)$ & $1.386(0.038)$ & $1.404(0.047)$ & $1.410(0.032)$ & $1.412(0.047)$ & $1.397(0.040)$ & $1.389(0.041)$ \\
\hline \multirow[t]{3}{*}{ WW } & Of & $0.970(0.026)$ & $0.969(0.025)$ & $0.973(0.026)$ & $0.990(0.015)$ & $0.972(0.015)$ & $0.961(0.022)$ & $0.988(0.019)$ & $0.978(0.027)$ & $0.985(0.025)$ & $0.982(0.022)$ & $0.977(0.024)$ \\
\hline & $\mathrm{Ov}$ & $0.946(0.023)$ & $0.940(0.025)$ & $0.946(0.023)$ & $0.970(0.029)$ & $0.947(0.027)$ & $0.942(0.026)$ & $0.970(0.023)$ & $0.948(0.033)$ & $0.982(0.023)$ & $0.947(0.031)$ & $0.954(0.029)$ \\
\hline & $\mathrm{Tt}$ & $0.947(0.028)$ & $0.953(0.031)$ & $0.935(0.030)$ & $0.954(0.027)$ & $0.948(0.022)$ & $0.943(0.026)$ & $0.966(0.021)$ & $0.959(0.029)$ & $0.971(0.023)$ & $0.960(0.027)$ & $0.953(0.028)$ \\
\hline \multirow[t]{3}{*}{ HW } & Of & $0.932(0.018)$ & $0.933(0.017)$ & $0.925(0.019)$ & $0.945(0.016)$ & $0.927(0.019)$ & $0.913(0.023)$ & $0.946(0.015)$ & $0.931(0.019)$ & $0.946(0.023)$ & $0.944(0.021)$ & $0.934(0.021)$ \\
\hline & $\mathrm{Ov}$ & $0.866(0.036)$ & $0.883(0.021)$ & $0.870(0.023)$ & $0.887(0.028)$ & $0.863(0.027)$ & $0.867(0.025)$ & $0.885(0.022)$ & $0.876(0.029)$ & $0.903(0.029)$ & $0.876(0.027)$ & $0.877(0.029)$ \\
\hline & $\mathrm{Tt}$ & $0.871(0.019)$ & $0.876(0.032)$ & $0.862(0.029)$ & $0.869(0.025)$ & $0.876(0.022)$ & $0.871(0.022)$ & $0.888(0.024)$ & $0.883(0.024)$ & $0.894(0.032)$ & $0.880(0.027)$ & $0.877(0.027)$ \\
\hline \multirow[t]{3}{*}{ FW } & Of & $0.402(0.016)$ & $0.405(0.015)$ & $0.390(0.018)$ & $0.423(0.012)$ & $0.402(0.012)$ & $0.388(0.016)$ & $0.416(0.014)$ & $0.399(0.014)$ & $0.411(0.016)$ & $0.413(0.013)$ & $0.405(0.018)$ \\
\hline & $\mathrm{Ov}$ & $0.369(0.014)$ & $0.386(0.015)$ & $0.370(0.012)$ & $0.399(0.014)$ & $0.376(0.015)$ & $0.373(0.013)$ & $0.386(0.012)$ & $0.376(0.015)$ & $0.399(0.013)$ & $0.384(0.015)$ & $0.382(0.017)$ \\
\hline & $\mathrm{Tt}$ & $0.371(0.017)$ & $0.387(0.016)$ & $0.371(0.014)$ & $0.386(0.015)$ & $0.385(0.012)$ & $0.374(0.011)$ & $0.392(0.016)$ & $0.378(0.016)$ & $0.394(0.017)$ & $0.390(0.015)$ & $0.383(0.017)$ \\
\hline \multirow[t]{3}{*}{ TL/WL } & Of & $0.694(0.021)$ & $0.702(0.017)$ & $0.692(0.018)$ & $0.699(0.021)$ & $0.698(0.017)$ & $0.687(0.018)$ & $0.690(0.019)$ & $0.696(0.017)$ & $0.699(0.024)$ & $0.697(0.014)$ & $0.695(0.019)$ \\
\hline & $\mathrm{Ov}$ & $0.690(0.019)$ & $0.707(0.015)$ & $0.683(0.016)$ & $0.698(0.017)$ & $0.695(0.021)$ & $0.697(0.019)$ & $0.698(0.019)$ & $0.695(0.017)$ & $0.703(0.017)$ & $0.692(0.016)$ & $0.696(0.018)$ \\
\hline & $\mathrm{Tt}$ & $0.677(0.016)$ & $0.692(0.024)$ & $0.671(0.015)$ & $0.686(0.014)$ & $0.693(0.017)$ & $0.678(0.020)$ & $0.681(0.019)$ & $0.684(0.014)$ & $0.689(0.024)$ & $0.678(0.018)$ & $0.683(0.019)$ \\
\hline
\end{tabular}

(b) Females

\begin{tabular}{|c|c|c|c|c|c|c|c|c|c|c|c|}
\hline \multirow[t]{3}{*}{ TL } & $1.075(0.027)$ & $1.092(0.029)$ & $1.080(0.030)$ & $1.101(0.027)$ & $1.066(0.020)$ & $1.069(0.030)$ & $1.106(0.024)$ & $1.089(0.029)$ & $1.113(0.016)$ & $1.098(0.028)$ & $1.089(0.030)$ \\
\hline & $1.031(0.038)$ & $1.026(0.038)$ & $1.007(0.039)$ & $1.052(0.036)$ & $1.025(0.034)$ & $1.005(0.033)$ & $1.032(0.036)$ & $1.002(0.036)$ & $1.064(0.037)$ & $1.015(0.035)$ & $1.026(0.040)$ \\
\hline & $0.998(0.052)$ & $1.014(0.031)$ & $1.014(0.036)$ & $1.035(0.045)$ & $1.018(0.032)$ & $1.022(0.031)$ & $1.027(0.042)$ & $1.021(0.029)$ & $1.048(0.050)$ & $1.009(0.050)$ & $1.021(0.042)$ \\
\hline \multirow[t]{3}{*}{ WL } & $1.538(0.033)$ & $1.537(0.033)$ & $1.533(0.026)$ & $1.556(0.041)$ & $1.519(0.034)$ & $1.534(0.037)$ & $1.578(0.035)$ & $1.536(0.047)$ & $1.569(0.041)$ & $1.553(0.040)$ & $1.545(0.040)$ \\
\hline & $1.467(0.034)$ & $1.453(0.048)$ & $1.441(0.036)$ & $1.504(0.049)$ & $1.474(0.047)$ & $1.445(0.042)$ & $1.475(0.048)$ & $.441(0.039)$ & $1.496(0.043)$ & $1.455(0.045)$ & $1.465(0.048)$ \\
\hline & $1.462(0.045)$ & $1.467(0.047)$ & $1.462(0.037)$ & $1.496(0.050)$ & $1.469(0.035)$ & & & & & & \\
\hline \multirow[t]{3}{*}{ WW } & $1.039(0.023)$ & $1.041(0.031)$ & $1.050(0.017)$ & $1.056(0.020)$ & $1.024(0.026)$ & $1.026(0.025)$ & $1.060(0.016)$ & 32) & 029) & $1.052(0.023)$ & $1.044(0.028)$ \\
\hline & $\mathrm{Ov}$ & & & & & & & & & & \\
\hline & $997(0.046)$ & .037) & 5) & & & & & & & & \\
\hline \multirow[t]{3}{*}{ HW } & $.974(0.014)$ & $0.977(0.019)$ & $0.967(0.018)$ & $0.990(0.019)$ & .018) & & $8(0.022)$ & & & & .024) \\
\hline & $\mathrm{Ov}$ & & & & & & & & & & \\
\hline & $\mathrm{Tt}$ & 27) & & 2) & & & & & & & \\
\hline \multirow[t]{3}{*}{ FW } & $0.439(0.014)$ & $0.441(0.017)$ & $0.421(0.013)$ & $0.456(0.015)$ & $0.425(0.013)$ & & $0.451(0.016)$ & & & & $(0.019)$ \\
\hline & $\mathrm{Ov}$ & & & & & & & & & & \\
\hline & $\mathrm{Tt}$ & 0.415 & & 18) & & & & & & 17) & $.017)$ \\
\hline \multirow[t]{3}{*}{ TL/WL } & $0.700(0.020)$ & $0.710(0.016)$ & $0.705(0.017)$ & $0.708(0.018)$ & $0.702(0.017)$ & $0.697(0.017)$ & $0.701(0.016)$ & $0.709(0.018)$ & $0.710(0.017)$ & $0.707(0.016)$ & $0.705(0.017)$ \\
\hline & $0.703(0.019)$ & & & & & & & & & & \\
\hline & $0.682(0.022)$ & $0.692(0.016)$ & $0.694(0.022)$ & $0.692(0.014)$ & $0.692(0.014)$ & $0.690(0.019)$ & $0.691(0.013)$ & $0.690(0.018)$ & $0.703(0.015)$ & $0.679(0.014)$ & $0.691(0.018)$ \\
\hline
\end{tabular}

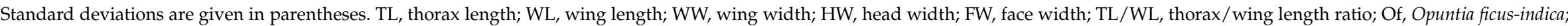
Ov, Opuntia vulgaris; Tt, Trichocereus terschekii. 